\title{
A Gauge Transformation between Ragnisco-Tu Hierarchy and a Related Lattice Hierarchy
}

\author{
Yuqing Liu1 ${ }^{*}$, Chao Hu1 ${ }^{1}$ Juan Dai ${ }^{2}$ \\ ${ }^{1}$ School of Physics \& Mathematics, Changzhou University, Changzhou, China \\ ${ }^{2}$ Information and Technique College of Changzhou, Changzhou, China \\ Email: ${ }^{*}$ yqmail321@163.com
}

Received 6 September 2015; accepted 25 October 2015; published 28 October 2015

Copyright (C) 2015 by authors and Scientific Research Publishing Inc.

This work is licensed under the Creative Commons Attribution International License (CC BY). http://creativecommons.org/licenses/by/4.0/

\section{(c) (i) Open Access}

\begin{abstract}
A new lattice hierarchy related to Ragnisco-Tu equation is proposed and its gauge equivalence to Ragnisco-Tu equation is proven. As an application of gauge transformation, we construct Darboux transformation (DT) of this new equation through DT of Ragnisco-Tu equation. An explicit exact solution is presented as an example.
\end{abstract}

\section{Keywords}

\section{Ragnisco-Tu Hierarchy, Gauge Transformation, Transfer Operator, Darboux Transformation}

\section{Introduction}

Nonlinear integrable equations usually have some marvellous properties such as Hamilton structure and infinitely many conservation laws. There are close connections between many of these equations. For instance, the cerebrated KdV equation, modified KdV equation, and nonlinear Schrödinger equation are reduction of AKNS system. Boussinesq equation and derivative nonlinear Schrödinger equation are linked to the constraint of KP equation (c.f. [1]). Jaulent-Miodek equation, Kaup-Newell equation, Levi equation and Heisenberg equation were found to be equivalent to AKNS equation [1]-[6]. The relation between two equations plays an important role, which makes one tackle with relatively unfamiliar equations through relatively familiar equations. However, in a general survey, there is comparatively less research on relatedness of lattice soliton equations than that of continuous soliton equations. One of the reasons behind this actuality is the lack of related example. In this paper we put forward a pair of nonlinear integrable lattice equations and investigate some relations such as gauge

"Corresponding author.

How to cite this paper: Liu, Y.Q., Hu, C. and Dai, J. (2015) A Gauge Transformation between Ragnisco-Tu Hierarchy and a Related Lattice Hierarchy. Journal of Applied Mathematics and Physics, 3, 1282-1294.

http://dx.doi.org/10.4236/jamp.2015.310157 
equivalence relation and Darboux transformation between them. Utilizing the relation which has been found, we will obtain an exact solution of equation.

Ragnisco-Tu equation [7] [8]

$$
Q_{n t}=E Q_{n}-R_{n} Q_{n}^{2}, R_{n t}=-E^{-1} R_{n}+R_{n}^{2} Q_{n},
$$

is an integrable lattice soliton equation. Ref. [8] discussed its Hamilton structure, and proved that its continuous limit may result in AKNS system. Ref. [9] obtained its inverse scattering transformation and exact solution. Ref. [10]-[12] researched more general problems, and studied Hamilton structure and Darboux transformation and geometric algerba solutions. Ragnisco-Tu equation has spectral problem [7]

$$
E \varphi_{n}=M \varphi_{n}, M=\left(\begin{array}{cc}
\lambda+R_{n} Q_{n} & Q_{n} \\
R_{n} & 1
\end{array}\right),
$$

where $\varphi_{n}=\left(\varphi_{1 n}, \varphi_{2 n}\right)^{\mathrm{T}}, E$ means a shift of space variable $n$, subscript $t$ denotes partial derivative with respect to time $t, \lambda$ is spectral parameter and $Q_{n}, R_{n}$ are potential functions. The derivation of equation hierarchy will be given in Section 2. This spectral problem can be generalized to

$$
E \psi_{n}=M^{\prime} \psi_{n}, \psi_{n}=\left(\psi_{1 n}, \psi_{2 n}\right)^{\mathrm{T}}, M^{\prime}=\left(\begin{array}{cc}
\lambda+(\lambda+1) q_{n} r_{n} & (\lambda+1) q_{n} \\
r_{n} & 1
\end{array}\right) .
$$

This method for constructing new lattice equation was first used in modified Toda equation [13] [14]. Surprisingly, equations obtained via these two spectral equations are equivalent, but this feature does not appear on Toda equation. On this basis, we further discuss Darboux transformation of them. With the help of gauge transformation and Darboux transformation of Ragnisco-Tu equation, we get a Darboux transformation of new equation, which is complex and difficult to construct directly.

This paper is organized as follows: in Section 2 and Section 3, we deduce the general hierarchies of Ragnisco-Tu and related generalized lattice hierarchy respectively. In Section 4, we derive a gauge transformation and transfer operator of two hierarchies. Section 5 will contribute to the Darboux transformation of two equations. Finally, in Section 6, a conclusion is presented.

\section{The Derivation of Ragnisco-Tu Hierarchy}

The derivation of Ragnisco-Tu hierarchy can be referred to [8] [9], but for completeness we still give a concise version.

Consider time evolution corresponding to (1.2)

$$
\varphi_{n, t}=N \varphi_{n}, N=\left(\begin{array}{cc}
A_{n} & B_{n} \\
C_{n} & D_{n}
\end{array}\right),
$$

discrete zero curvature equation results in following equalities directly

$$
\begin{gathered}
\lambda_{t}+\left(R_{n} Q_{n}\right)_{t}=\left(\lambda+R_{n} Q_{n}\right)(E-1) A_{n}+R_{n} E B_{n}-Q_{n} C_{n}, \\
Q_{n, t}=Q_{n}\left(E A_{n}-D_{n}\right)+E B_{n}-\left(\lambda+R_{n} Q_{n}\right) B_{n}, \\
R_{n, t}=\left(\lambda+R_{n} Q_{n}\right) E C_{n}-C_{n}+R_{n}\left(E D_{n}-A_{n}\right), \\
0=Q_{n} E C_{n}-R_{n} B_{n}+(E-1) D_{n} .
\end{gathered}
$$

From these equations we draw out relations between related quantities

$$
\begin{gathered}
D=(E-1)^{-1}\left(R_{n} B_{n}-Q_{n} E C_{n}\right)+d_{0}, \\
A_{n}+D_{n}=n \lambda^{-1} \lambda_{t}+a_{0},
\end{gathered}
$$

where $(E-1)^{-1}$ defined as $(E-1)^{-1} f_{n}=\frac{1}{2}\left(-\sum_{k=n}^{\infty}+\sum_{k=-\infty}^{n-1}\right) f_{k}$, and $d_{0}, a_{0}$ are constants independent of variable $n$. Let 


$$
\left(\begin{array}{c}
E C_{n} \\
B_{n}
\end{array}\right)=\sum_{j=0}^{k}\left(\begin{array}{c}
E C_{n j} \\
b_{n j}
\end{array}\right) \lambda^{k-j},
$$

the relations (2.2)-(2.5) can be written as

$$
\left(\begin{array}{l}
Q_{n} \\
R_{n}
\end{array}\right)_{t}=\sum_{j=0}^{k} L_{1}\left(\begin{array}{c}
E c_{n j} \\
b_{n j}
\end{array}\right) \lambda^{k-j}+\left(\begin{array}{c}
\left(a_{0}-2 d_{0}\right) Q_{n} \\
-\left(a_{0}-2 d_{0}\right) R_{n}
\end{array}\right)-\sum_{j=-1}^{k-1} L_{2}\left(\begin{array}{c}
E c_{n, j+1} \\
b_{n, j+1}
\end{array}\right) \lambda^{k-j}+\left(\begin{array}{c}
(n+1) Q_{n} \lambda^{-1} \lambda_{t} \\
-n R_{n} \lambda^{-1} \lambda_{t}
\end{array}\right),
$$

where operators $L_{i}, i=1,2$ are defined as

$$
\begin{gathered}
L_{1}=\left(\begin{array}{cc}
Q_{n} \Delta Q_{n} & -Q_{n} \Delta R_{n}+E-R_{n} Q_{n} \\
R_{n} Q_{n}-E^{-1}-R_{n} \Delta Q_{n} & R_{n} \Delta R_{n}
\end{array}\right), \\
L_{2}=\left(\begin{array}{cc}
0 & 1 \\
-1 & 0
\end{array}\right), \Delta=(E+1)(E-1)^{-1} .
\end{gathered}
$$

Giving boundary condition

$$
\left(\begin{array}{cc}
A_{n} & B_{n} \\
C_{n} & D_{n}
\end{array}\right)_{Q_{n}=R_{n}=0}=\left(\begin{array}{cc}
\frac{1}{2} \lambda^{k+1} & 0 \\
0 & -\frac{1}{2} \lambda^{k+1}
\end{array}\right)
$$

and taking $\lambda_{t}=0, a_{0}-2 d_{0}=\lambda^{k+1}$ we may deduce the iso-spectral hierarchy as follows:

$$
\left(\begin{array}{l}
Q_{n} \\
R_{n}
\end{array}\right)_{t}=\left(L_{1} L_{2}^{-1}\right)^{k+1}\left(\begin{array}{c}
Q_{n} \\
-R_{n}
\end{array}\right)
$$

The case of $k=0$ just gives Ragnisco-Tu Equation (1.1).

If the boundary condition is given as

$$
\left(\begin{array}{cc}
A_{n} & B_{n} \\
C_{n} & D_{n}
\end{array}\right)_{Q_{n}=R_{n}=0}=\left(\begin{array}{cc}
\frac{n(\lambda+1) \lambda^{k}}{2} & 0 \\
0 & -\frac{n(\lambda+1) \lambda^{k}}{2}
\end{array}\right)
$$

and $\lambda_{t}=(\lambda+1) \lambda^{k+1}, a_{0}-2 d_{0}=0$ we get non-iso-spectral Ragnisco-Tu hierarchy

$$
\left(\begin{array}{c}
Q_{n} \\
R_{n}
\end{array}\right)_{t}=\left(L_{1} L_{2}^{-1}+I\right)\left(L_{1} L_{2}^{-1}\right)^{k}\left(\begin{array}{c}
(n+1) Q_{n} \\
-n R_{n}
\end{array}\right)
$$

where $I$ is an identity operator. In more general case, Ragnisco-Tu hierarchy is expressed by

Lemma 2.1. If $\omega(\lambda), \rho(\lambda)$ are the polynomials of $\lambda$ with degree $k, k+1$ respectively, $\lambda_{t}=(\lambda+1) \lambda \omega(\lambda)$, and the boundary condition is as follows

$$
\left(\begin{array}{cc}
A_{n} & B_{n} \\
C_{n} & D_{n}
\end{array}\right)_{Q_{n}=R_{n}=0}=\left(\begin{array}{cc}
\frac{n(\lambda+1)}{2} \omega(\lambda)+\frac{1}{2} \rho(\lambda) & 0 \\
0 & -\frac{n(\lambda+1)}{2} \omega(\lambda)-\frac{1}{2} \rho(\lambda)
\end{array}\right),
$$

then general Ragnisco-Tu hierarchy adopts the from

$$
\left(\begin{array}{l}
Q_{n} \\
R_{n}
\end{array}\right)_{t}=(L+I) \omega(L)\left(\begin{array}{c}
(n+1) Q_{n} \\
-n R_{n}
\end{array}\right)+\rho(L)\left(\begin{array}{c}
Q_{n} \\
-R_{n}
\end{array}\right), L=L_{1} L_{2}^{-1} .
$$

\section{A New Lattice Hierarchy Related to the Ragnisco-Tu Hierarchy}

With regard to generalized spectral problem (1.3), introduce the time evolution 


$$
\psi_{n, t}=N^{\prime} \psi_{n}, \quad N^{\prime}=\left(\begin{array}{cc}
A_{n}^{\prime} & (\lambda+1) B_{n}^{\prime} \\
C_{n}^{\prime} & D_{n}^{\prime}
\end{array}\right) .
$$

Then from discrete zero curvature equation, we have

$$
\begin{gathered}
(\lambda+1)\left(q_{n} r_{n}\right)_{t}=\left[\lambda+(\lambda+1) q_{n} r_{n}\right](E-1) A_{n}^{\prime}+(\lambda+1)\left(r_{n} E B_{n}^{\prime}-q_{n} C_{n}^{\prime}\right)-\left(1+q_{n} r_{n}\right) \lambda_{t}, \\
(\lambda+1) q_{n, t}=(\lambda+1) q_{n}\left(E A_{n}^{\prime}-D_{n}^{\prime}\right)+(\lambda+1)\left[E B_{n}^{\prime}-\lambda B_{n}^{\prime}-(\lambda+1) q_{n} r_{n} B_{n}^{\prime}\right]-q_{n} \lambda_{t}, \\
r_{n, t}=r\left(E D_{n}^{\prime}-A_{n}^{\prime}\right)-C_{n}^{\prime}+\left[\lambda+(\lambda+1) q_{n} r_{n}\right] E C_{n}^{\prime}, \\
0=(\lambda+1)\left(q_{n} E C_{n}^{\prime}-r_{n} B_{n}^{\prime}\right)+(E-1) D_{n}^{\prime} .
\end{gathered}
$$

It is ease to know that there only have three independent equations, for instance, (3.2b), (3.2c), (3.2d). Now, from them we work out

$$
A_{n}^{\prime}+D_{n}^{\prime}=n \lambda^{-1} \lambda_{t}+a_{0}^{\prime}
$$

and

$$
D_{n}^{\prime}=-(\lambda+1)(E-1)^{-1}\left(q_{n},-r_{n}\right)\left(\begin{array}{c}
E C_{n}^{\prime} \\
B_{n}^{\prime}
\end{array}\right)+d_{0}^{\prime},
$$

where $a_{0}^{\prime}, d_{0}^{\prime}$ are independent of $n$. Introducing two operators $L_{1}^{\prime}, L_{2}^{\prime}$

$$
\begin{gathered}
L_{1}^{\prime}=\left(\begin{array}{cc}
q_{n} \Delta q_{n} & -q_{n} \Delta r_{n}-q_{n} r_{n}+E \\
-r_{n} \Delta q_{n}+q_{n} r_{n}-E^{-1} & r_{n} \Delta r_{n}
\end{array}\right), \\
L_{2}^{\prime}=\left(\begin{array}{cc}
-q_{n} \Delta q_{n} & q_{n} \Delta r_{n}+1+q_{n} r_{n} \\
r_{n} \Delta q_{n}-1-q_{n} r_{n} & -r_{n} \Delta r_{n}
\end{array}\right),
\end{gathered}
$$

we get matrix form

$$
\left(\begin{array}{c}
q_{n} \\
r_{n}
\end{array}\right)_{t}=L_{1}^{\prime}\left(\begin{array}{c}
E C_{n}^{\prime} \\
B_{n}^{\prime}
\end{array}\right)-\lambda L_{2}^{\prime}\left(\begin{array}{c}
E C_{n}^{\prime} \\
B_{n}^{\prime}
\end{array}\right)-\left(\begin{array}{c}
q_{n} \\
0
\end{array}\right) \frac{\lambda_{t}}{\lambda+1}+\left(a_{0}^{\prime}-2 d_{0}^{\prime}\right)\left(\begin{array}{c}
q_{n} \\
-r_{n}
\end{array}\right)+\lambda^{-1} \lambda_{t}\left(\begin{array}{c}
(n+1) q_{n} \\
-n r_{n}
\end{array}\right) .
$$

Set

$$
\left(\begin{array}{c}
E C_{n}^{\prime} \\
B_{n}^{\prime}
\end{array}\right)=\sum_{j=0}^{k}\left(\begin{array}{c}
E c_{n, j}^{\prime} \\
b_{n, j}^{\prime}
\end{array}\right) \lambda^{k-j},
$$

the general lattice hierarchy (called generalized Ragnisco-Tu hierarchy) is deduced in

Lemma 3.1. Let $\tilde{\omega}(\lambda)$ and $\tilde{\rho}(\lambda)$ be the polynomials of $\lambda$ with degree $k$ and $(k+1)$ respectively, take $\lambda_{t}=\lambda(\lambda+1) \tilde{\omega}(\lambda), a_{0}^{\prime}-2 d_{0}^{\prime}=\tilde{\rho}(\lambda)$, then under boundary condition

$$
\left.\left(\begin{array}{cc}
A_{n}^{\prime} & (\lambda+1) B_{n}^{\prime} \\
C_{n}^{\prime} & D_{n}^{\prime}
\end{array}\right)\right|_{q_{n}=r_{n}=0}=\left(\begin{array}{cc}
\frac{n(\lambda+1)}{2} \tilde{\omega}(\lambda)+\frac{1}{2} \tilde{\rho}(\lambda) & 0 \\
0 & -\frac{n(\lambda+1)}{2} \tilde{\omega}(\lambda)-\frac{1}{2} \tilde{\rho}(\lambda)
\end{array}\right),
$$

the generalized Ragnisco-Tu hierarchy is

$$
\left(\begin{array}{l}
q_{n} \\
r_{n}
\end{array}\right)_{t}=L^{\prime} \tilde{\omega}\left(L^{\prime}\right)\left(\begin{array}{c}
n q_{n} \\
-n r_{n}
\end{array}\right)+\tilde{\omega}\left(L^{\prime}\right)\left(\begin{array}{c}
(n+1) q_{n} \\
-n r_{n}
\end{array}\right)+\tilde{\rho}\left(L^{\prime}\right)\left(\begin{array}{c}
q_{n} \\
-r_{n}
\end{array}\right), L^{\prime}=L_{1}^{\prime}\left(L_{2}^{\prime-1}\right) .
$$

Especially when $\tilde{\omega}(\lambda)=0$ and $\tilde{\rho}(\lambda)=\lambda^{k+1}$, it is the iso-spectral hierarchy

$$
\left(\begin{array}{c}
q_{n} \\
r_{n}
\end{array}\right)_{t}=L^{k+1}\left(\begin{array}{c}
q_{n} \\
-r_{n}
\end{array}\right)
$$


The first one $(k=0)$ is

$$
\left(\begin{array}{l}
q_{n} \\
r_{n}
\end{array}\right)_{t}=\left(\begin{array}{c}
E \frac{q_{n}}{1+q_{n} r_{n}}-\frac{q_{n}^{2} r_{n}}{1+q_{n} r_{n}} \\
-E^{-1} \frac{r_{n}}{1+q_{n} r_{n}}+\frac{q_{n} r_{n}^{2}}{1+q_{n} r_{n}}
\end{array}\right)
$$

If we take $\tilde{\omega}(\lambda)=\lambda^{k}, \tilde{\rho}(\lambda)=0$, then we get the non-iso-spectral hierarchy as follows

$$
\left(\begin{array}{l}
q_{n} \\
r_{n}
\end{array}\right)_{t}=\left(L^{\prime}\right)^{k+1}\left(\begin{array}{c}
n q_{n} \\
-n r_{n}
\end{array}\right)+\left(L^{\prime}\right)^{k}\left(\begin{array}{c}
(n+1) q_{n} \\
-n r_{n}
\end{array}\right) .
$$

The first one $(k=0)$ is

$$
\left(\begin{array}{l}
q_{n} \\
r_{n}
\end{array}\right)=\left(\begin{array}{c}
(n+1) E \frac{q_{n}}{1+q_{n} r_{n}}+\frac{(n+1) q_{n}^{2} r_{n}}{1+q_{n} r_{n}} \\
-(n-1) E^{-1} \frac{r_{n}}{1+q_{n} r_{n}}-\frac{n r_{n}}{1+q_{n} r_{n}}
\end{array}\right) .
$$

Proof. Expanding (3.7) we have

$$
\begin{aligned}
\left(\begin{array}{c}
q_{n} \\
r_{n}
\end{array}\right)_{t}= & \sum_{j=0}^{k} L_{1}^{\prime}\left(\begin{array}{c}
E c_{n, j}^{\prime} \\
b_{n, j}^{\prime}
\end{array}\right) \lambda^{k-j}-\sum_{j=-1}^{k-1} L_{2}^{\prime}\left(\begin{array}{c}
E c_{n, j+1}^{\prime} \\
b_{n, j+1}^{\prime}
\end{array}\right) \lambda^{k-j}+\sum_{j=-1}^{k-1} \tilde{\omega}_{j+1}\left(\begin{array}{c}
n q_{n} \\
-n r_{n}
\end{array}\right) \lambda^{k-j} \\
& +\sum_{j=0}^{k} \tilde{\omega}_{j}\left(\begin{array}{c}
(n+1) q_{n} \\
-n r_{n}
\end{array}\right) \lambda^{k-j}+\sum_{j=-1}^{k} \tilde{\rho}_{j+1}\left(\begin{array}{c}
q_{n} \\
r_{n}
\end{array}\right) \lambda^{k-j} .
\end{aligned}
$$

Equating the coefficients of power of $\lambda$ leads to

$$
\begin{gathered}
\left(\begin{array}{c}
q_{n, t} \\
r_{n, t}
\end{array}\right)=L_{1}^{\prime}\left(\begin{array}{c}
E c_{n, k}^{\prime} \\
b_{n, k}^{\prime}
\end{array}\right)+\tilde{\omega}_{k}\left(\begin{array}{c}
(n+1) q_{n} \\
-n r_{n}
\end{array}\right)+\tilde{\rho}_{k+1}\left(\begin{array}{c}
q_{n} \\
r_{n}
\end{array}\right), \\
L_{2}^{\prime}\left(\begin{array}{c}
E c_{n, j+1}^{\prime} \\
b_{n, j+1}^{\prime}
\end{array}\right)=L_{1}^{\prime}\left(\begin{array}{c}
E c_{n, j}^{\prime} \\
b_{n, j}^{\prime}
\end{array}\right)+\tilde{\omega}_{j+1}\left(\begin{array}{c}
n q_{n} \\
-n r_{n}
\end{array}\right)+\tilde{\omega}_{j}\left(\begin{array}{c}
(n+1) q_{n} \\
-n r_{n}
\end{array}\right)+\tilde{\rho}_{j+1}\left(\begin{array}{c}
q_{n} \\
-r_{n}
\end{array}\right), j=0,1, \cdots, k-1, \\
L_{2}^{\prime}\left(\begin{array}{c}
E c_{n, 0}^{\prime} \\
b_{n, 0}^{\prime}
\end{array}\right)=\tilde{\omega}_{0}\left(\begin{array}{c}
n q_{n} \\
-n r_{n}
\end{array}\right)+\tilde{\rho}_{0}\left(\begin{array}{c}
q_{n} \\
-r_{n}
\end{array}\right) .
\end{gathered}
$$

Through mathematical induction we get the recursion relation

$$
\begin{aligned}
\left(\begin{array}{c}
E c_{n, j}^{\prime} \\
b_{n, j}^{\prime}
\end{array}\right)= & \left(\left(L_{2}^{\prime}\right)^{-1} L_{1}^{\prime}\right)^{j}\left(\begin{array}{c}
E c_{n, 0}^{\prime} \\
b_{n, 0}^{\prime}
\end{array}\right)+\sum_{s=0}^{j-1}\left(\left(L_{2}^{\prime}\right)^{-1} L_{1}^{\prime}\right)^{s}\left(L_{2}^{\prime}\right)^{-1} \tilde{\rho}_{j-s}\left(\begin{array}{c}
q_{n} \\
-r_{n}
\end{array}\right) \\
& +\sum_{s=0}^{j-1} \tilde{\omega}_{j-s}\left(\left(L_{2}^{\prime}\right)^{-1} L_{1}^{\prime}\right)^{s}\left(L_{2}^{\prime}\right)^{-1}\left(\begin{array}{c}
n q_{n} \\
-n r_{n}
\end{array}\right)+\sum_{s=0}^{j-1} \tilde{\omega}_{j-1-s}\left(\left(L_{2}^{\prime}\right)^{-1} L_{1}^{\prime}\right)^{s}\left(L_{2}^{\prime}\right)^{-1}\left(\begin{array}{c}
(n+1) q_{n} \\
-n r_{n}
\end{array}\right) .
\end{aligned}
$$

From it the conclusion of Lemma 3.1 is got.

\section{A Gauge Transformation and Transfer Operator between the Ragnisco-Tu Hierarchy and Generalized Ragnisco-Tu Hierarchy}

In this section we will give the conclusion about gauge transformation and transfer operator between the Ragnisco-Tu hierarchy and generalized Ragnisco-Tu hierarchy.

Theorem 4.1. There exists a gauge transformation changing Lax pair of generalized Ragnisco-Tu hierarchy (1.3), (3.1) into Lax pair of Ragnisco-Tu hierarchy: 


$$
\varphi_{n}=T \psi_{n}, T=\left(\begin{array}{cc}
E^{-1} \mu_{n} & \mu_{n} q_{n} \\
0 & E^{-1} \mu_{n}^{-1}
\end{array}\right), \mu_{n}=\prod_{j=n+1}^{\infty}\left(1+q_{j} r_{j}\right) .
$$

Further, potentials in (1.2) and those in (1.3) have the relations

$$
Q_{n}=\mu_{n}^{2} q_{n}+\mu_{n} E\left(\mu_{n} q_{n}\right), R_{n}=\frac{r_{n}}{\mu_{n} E^{-1} \mu_{n}} .
$$

When $\tilde{\omega}(\lambda)=\omega(\lambda), \quad \tilde{\rho}(\lambda)=\rho(\lambda)$, hierarchy (2.15) and hierarchy (3.9) satisfy

$$
\begin{aligned}
& L_{2}^{-1}\left[\left(\begin{array}{l}
Q_{n} \\
R_{n}
\end{array}\right)_{t}-(L+I) \omega(L)\left(\begin{array}{c}
(n+1) Q_{n} \\
-n R_{n}
\end{array}\right)+\rho(L)\left(\begin{array}{c}
Q_{n} \\
-R_{n}
\end{array}\right)\right] \\
& =\Omega\left(L_{2}^{\prime}\right)^{-1}\left[\left(\begin{array}{l}
q_{n} \\
r_{n}
\end{array}\right)_{t}-L^{\prime} \omega\left(L^{\prime}\right)\left(\begin{array}{c}
n q_{n} \\
-n r_{n}
\end{array}\right)+\omega\left(L^{\prime}\right)\left(\begin{array}{c}
(n+1) q_{n} \\
-n r_{n}
\end{array}\right)+\rho\left(L^{\prime}\right)\left(\begin{array}{c}
q_{n} \\
-r_{n}
\end{array}\right)\right],
\end{aligned}
$$

where $\Omega$ is transfer operator defined as

$$
\Omega=\left(\begin{array}{cc}
\mu_{n}^{-2} & 0 \\
\mu_{n}^{2} q_{n}^{2} & \mu_{n}^{2}(E+1)
\end{array}\right) .
$$

Proof. As gauge transformation, $T$ should satisfy

$$
(E T) M^{\prime}=M T \text {. }
$$

Set

$$
T=\left(\begin{array}{ll}
T_{1} & T_{2} \\
T_{3} & T_{4}
\end{array}\right)
$$

the entries of it must meet the following equations

$$
\begin{gathered}
\lambda\left(1+q_{n} r_{n}\right) E T_{1}+q_{n} r_{n} E T_{1}+r_{n} E T_{2}=\left(\lambda+Q_{n} R_{n}\right) T_{1}+Q_{n} T_{3}, \\
E T_{2}+(\lambda+1) q_{n} E T_{1}=\left(\lambda+R_{n} Q_{n}\right) T_{2}+Q_{n} T_{4}, \\
\lambda E T_{3}+(\lambda+1) q_{n} r_{n} E T_{3}+r_{n} E T_{4}=R_{n} T_{1}+T_{3}, \\
(\lambda+1) q_{n} E T_{3}+E T_{4}=R_{n} T_{2}+T_{4} .
\end{gathered}
$$

Notice that $T$ is independent of $\lambda$, its entries are determined easily.

Transformation matrix $T$ also changes time evolution (3.1) into (2.1). To justify this assertion, for a newly defined $\tilde{N}=\left(T_{t}+T N^{\prime}\right) T^{-1}$ or $\tilde{N}=S^{-1}\left(N^{\prime} S-S_{t}\right)$, where $S=T^{-1}$, we need to prove that equality $\tilde{N}=N$ is hold. A simple calculation shows us

$$
\tilde{N}=\left(\begin{array}{ll}
\tilde{N}_{11} & \tilde{N}_{12} \\
\tilde{N}_{21} & \tilde{N}_{22}
\end{array}\right)
$$

where

$$
\begin{gathered}
\tilde{N}_{11}=A_{n}^{\prime}+E^{-1}\left(\ln \mu_{n}\right)_{t}+C_{n}^{\prime} q_{n} /\left(1+q_{n} r_{n}\right), \\
\tilde{N}_{12}=\left(D_{n}^{\prime}-A_{n}^{\prime}\right) q_{n} \mu_{n} E^{-1} \mu_{n}+(\lambda+1) B_{n}^{\prime} E^{-1} \mu_{n}^{2}-C_{n}^{\prime} q_{n}^{2} \mu_{n}^{2} \\
+q_{n, t} \mu_{n} E^{-1} \mu_{n}+q_{n} \mu_{n} E^{-1} \mu_{n}\left(1-E^{-1}\right)\left(\ln \mu_{n}\right)_{t}, \\
\tilde{N}_{21}=C_{n}^{\prime} / E^{-1} \mu_{n}^{2}, \\
\tilde{N}_{22}=D_{n}^{\prime}-E^{-1}\left(\ln \mu_{n}\right)_{t}-C_{n}^{\prime} q_{n} /\left(1+q_{n} r_{n}\right) .
\end{gathered}
$$

It is evident that $\tilde{N}_{11}, \tilde{N}_{12}, \tilde{N}_{21}, \tilde{N}_{22}$ are the polynomial of $\lambda$ with degree $k+1, k, k, k+1$ respec- 
tively, which are the same as that of elements of $N$. In the meantime, the condition $q_{n}=r_{n}=0$ is equivalent to $Q_{n}=R_{n}=0$ and thus if permitting $\tilde{\omega}(\lambda)=\omega(\lambda), \tilde{\rho}(\lambda)=\rho(\lambda)$ then $\left.\tilde{N}\right|_{q_{n}=r_{n}=0}=\left.N\right|_{Q_{n}=R_{n}=0}$ is valid. On the other hand, we can verify directly that $M_{t}=(E \tilde{N}) M-M \tilde{N}$. Combining all discussed above we conclude that $\tilde{N}=N$. That is, the gauge transformation (4.1) conveys time evolution of (3.1) into that of (2.1).

Now we deduce transfer operator of two hierarchies. A dull calculation simplifies the expression of $\tilde{N}_{12}$ as

$$
\tilde{N}_{12}=\mu_{n}^{2}\left(E C_{n}^{\prime}\right) q_{n}^{2}+\mu_{n}^{2}(E+1) B_{n}^{\prime}+\frac{\lambda^{-1} \lambda_{t}}{\lambda+1} \mu_{n}^{2} q_{n}
$$

Thus we have

$$
\left(\begin{array}{c}
E c_{n j} \\
b_{n j}
\end{array}\right)=\left(\begin{array}{cc}
\mu_{n}^{-2} & 0 \\
\mu_{n}^{2} q_{n}^{2} & \mu_{n}^{2}(E+1)
\end{array}\right)\left(\begin{array}{c}
E c_{n j}^{\prime} \\
b_{n j}^{\prime}
\end{array}\right),
$$

where $j=0,1, \cdots, k-1$ for iso-spectral and $j=1,2, \cdots, k-1$ for non-iso-spectral.

Because of in the case of iso-spectral and non-iso-spectral, the following recursion formula always holds

$$
\left(\begin{array}{c}
E c_{n, j+1} \\
b_{n, j+1}
\end{array}\right)=L_{2}^{-1} L_{1}\left(\begin{array}{c}
E c_{n, j} \\
b_{n, j}
\end{array}\right), j=1,2, \cdots, k-1,
$$

(In the case of iso-spectral, $j=0,1, \cdots, k-1$ ),

$$
\left(\begin{array}{c}
E c_{n, j+1}^{\prime} \\
b_{n, j+1}^{\prime}
\end{array}\right)=\left(L_{2}^{\prime}\right)^{-1} L_{1}^{\prime}\left(\begin{array}{c}
E c_{n, j}^{\prime} \\
b_{n, j}^{\prime}
\end{array}\right), j=1,2, \cdots, k-1,
$$

(In the case of iso-spectral, $j=0,1, \cdots, k-1$ ), we can deduce transformation relation by substituting the above relations into (4.7)

$$
L_{2}^{-1} L_{1} \Omega=\Omega\left(\left(L_{2}^{\prime}\right)^{-1} L_{1}^{\prime}\right),
$$

where $\Omega$ is the transfer operator of two hierarchies. Comparing the coefficients of $\lambda^{0}$ in $B_{n}=\tilde{N}_{12}, C_{n}=\tilde{N}_{21}$ we have

$$
\left(\begin{array}{c}
E c_{n k} \\
b_{n k}
\end{array}\right)=\Omega\left(\begin{array}{c}
E c_{n k}^{\prime} \\
b_{n k}^{\prime}
\end{array}\right) .
$$

According to the derivation expressions of iso-spectral and non-iso-spectral equation we arrive at the relation of two hierarchies immediately

$$
L_{2}^{-1}\left(\begin{array}{l}
Q_{n} \\
R_{n}
\end{array}\right)_{t}=\Omega\left(L_{2}^{\prime}\right)^{-1}\left(\begin{array}{l}
q_{n} \\
r_{n}
\end{array}\right) .
$$

When we focus our attention on the iso-spectral case, (4.7) holds for $j=0$. That is

$$
\left(\begin{array}{l}
R_{n} \\
Q_{n}
\end{array}\right)=\Omega\left(\begin{array}{c}
\frac{r_{n}}{1+q_{n} r_{n}} \\
\frac{q_{n}}{1+q_{n} r_{n}}
\end{array}\right),
$$

which can be verified readily. When we concern about the non-iso-spectral case, (4.7) holds for $j=1$, the verification is not so easy. To get the equation

$$
\left(\begin{array}{c}
E c_{n 1} \\
b_{n 1}
\end{array}\right)=\Omega\left(\begin{array}{c}
E c_{n 1}^{\prime} \\
b_{n 1}^{\prime}
\end{array}\right)
$$

we first prove

$$
\Omega\left(L_{2}^{\prime}\right)^{-1}\left(\begin{array}{c}
(n+1) q_{n} \\
-n r_{n}
\end{array}\right)=L_{2}^{-1} L_{1}\left(\begin{array}{c}
0 \\
\mu_{n}^{2} q_{n}
\end{array}\right)+L_{2}^{-1}\left(\begin{array}{c}
(n+1) Q_{n} \\
-n R_{n}
\end{array}\right) .
$$


Denote

$$
\text { l.h.s }=\left(\begin{array}{l}
\text { leftline } 1 \\
\text { leftline2 }
\end{array}\right), \text { r.h.s }=\left(\begin{array}{c}
\text { rightline1 } \\
\text { rightline2 }
\end{array}\right) .
$$

It is ease to know "leftline $1=$ rightline1". As for "line 2 ” we have

$$
\begin{aligned}
\text { leftline } 2= & n \mu_{n}^{2} q_{n}+\mu_{n} E\left(\mu_{n} q_{n}\right)+\frac{\mu_{n}^{2} q_{n}}{1+q_{n} r_{n}}+\mu_{n}\left(E \mu_{n}\right)\left(E n q_{n}\right) \\
& -\mu_{n}^{2} E\left(\frac{q_{n}}{1+q_{n} r_{n}} \Delta \frac{q_{n} r_{n}}{1+q_{n} r_{n}}\right)-\mu_{n}^{2} q_{n} \Delta \frac{q_{n} r_{n}}{1+q_{n} r_{n}}
\end{aligned}
$$

and

$$
\begin{aligned}
\text { rightline } 2= & n \mu_{n}^{2} q_{n}+E\left(\mu_{n}^{2} q_{n}\right)+\frac{\mu_{n}^{2} q_{n}}{1+q_{n} r_{n}}-\mu_{n} E\left(\mu_{n} q_{n}\right) \frac{q_{n} r_{n}}{1+q_{n} r_{n}} \\
& +\mu_{n}\left(E \mu_{n}\right)\left(E n q_{n}\right)-\left(\mu_{n}^{2} q_{n}+\mu_{n} E\left(\mu_{n} q_{n}\right)\right) \Delta \frac{q_{n} r_{n}}{1+q_{n} r_{n}} .
\end{aligned}
$$

Their difference is

$$
\begin{aligned}
& \mu_{n} E\left(\mu_{n} q_{n}\right)-E\left(\mu_{n}^{2} q_{n}\right)+\mu_{n} E\left(\mu_{n} q_{n}\right) \frac{q_{n} r_{n}}{1+q_{n} r_{n}}-\mu_{n}^{2} E\left(\frac{q_{n}}{1+q_{n} r_{n}} \Delta \frac{q_{n} r_{n}}{1+q_{n} r_{n}}\right)+\mu_{n} E\left(\mu_{n} q_{n}\right) \Delta \frac{q_{n} r_{n}}{1+q_{n} r_{n}} \\
& =\mu_{n} E\left(\mu_{n} q_{n}\right)-E\left(\mu_{n}^{2} q_{n}\right)+\mu_{n} E\left(\mu_{n} q_{n}\right) \frac{q_{n} r_{n}}{1+q_{n} r_{n}}-\mu_{n} E\left(\mu_{n} q_{n}\right)(E+1) \frac{q_{n} r_{n}}{1+q_{n} r_{n}}=0 .
\end{aligned}
$$

The Equation (4.11) is proved.

On the other hand, through comparing the coefficients of $\lambda^{k}$ in $B_{n}=\tilde{N}_{12}, C_{n}=\tilde{N}_{21}$ we have

$$
\left(\begin{array}{c}
n R_{n} \\
(n+1) Q_{n}
\end{array}\right)=\Omega\left(\begin{array}{c}
\frac{n r_{n}}{1+q_{n} r_{n}} \\
\frac{n q_{n}}{1+q_{n} r_{n}}
\end{array}\right)+\left(\begin{array}{c}
0 \\
\mu_{n}^{2} q_{n}
\end{array}\right) .
$$

That is

$$
L_{2}^{-1}\left(\begin{array}{c}
(n+1) Q_{n} \\
-n R_{n}
\end{array}\right)=\Omega\left(L_{2}^{\prime}\right)^{-1}\left(\begin{array}{c}
n q_{n} \\
-n r_{n}
\end{array}\right)+\left(\begin{array}{c}
0 \\
\mu_{n}^{2} q_{n}
\end{array}\right)
$$

Using the recursion relations of $\left(\begin{array}{c}E c_{n j} \\ b_{n j}\end{array}\right)$ and $\left(\begin{array}{c}E c_{n j}^{\prime} \\ b_{n j}^{\prime}\end{array}\right)$ together with (4.11), (4.12) and the formula of transformation operator (4.8) we finally obtain the recursion relation of non-iso-spectral (4.10).

Finally, we consider relevancy of two hierarchy. The time part of (4.3) has given in (4.9). The following equation is deduced according to (4.13) and (4.8)

$$
L_{2}^{-1} L\left(\begin{array}{c}
(n+1) Q_{n} \\
-n R_{n}
\end{array}\right)=\Omega\left(L_{2}^{\prime}\right)^{-1}\left(\begin{array}{c}
n q_{n} \\
-n r_{n}
\end{array}\right)+\left(\begin{array}{c}
0 \\
\mu_{n}^{2} q_{n}
\end{array}\right) .
$$

Equation (4.11) together with above expression yields

$$
L_{2}^{-1}(L+I)\left(\begin{array}{c}
(n+1) Q_{n} \\
-n R_{n}
\end{array}\right)=\Omega\left(L_{2}^{\prime}\right)^{-1}\left[L^{\prime}\left(\begin{array}{c}
n q_{n} \\
-n r_{n}
\end{array}\right)+\left(\begin{array}{c}
(n+1) q_{n} \\
-n r_{n}
\end{array}\right)\right] .
$$

Through mathematical induction we can prove the part of $\omega$ in (4.3). The proof of part of $\rho$ is similar and it is much simpler. Thus we finish the theorem. 


\section{Darboux Transformation to Generalized Ragnisco-Tu Equation}

Darboux transformation is a very useful tool to obtain exact solutions of nonlinear integral equation. It plays role in every type of equations such as lattice equation, discrete equation and high dimensional integral equation [15]-[22]. However, the construction of DT of complex system may still encounter difficulty. Here, we will have the aid of gauge transformation to consider DT of generalized Ragnisco-Tu equation.

\subsection{Darboux Transformation to Ragnisco-Tu Equation}

Consider transformation

$$
\bar{\varphi}=H \varphi, H=\left(\begin{array}{cc}
\lambda+h_{1} & h_{2} \\
h_{3} & \lambda+h_{4}
\end{array}\right),
$$

where $h_{j}, j=1,2,3,4$ are independent of $\lambda$. We can see that it is a DT of Ragnisco-Tu hierarchy:

Lemma 5.1. (see also [12]) Suppose $h_{2}, h_{3} \rightarrow 0$ as $|n| \rightarrow \infty$, then aforementioned transformation (5.1) is DT of Ragnisco-Tu hierarchy expressed by Lemma 2.1 with $\omega(\lambda)=0, \rho(\lambda)=\lambda^{k+1}$. If $\bar{R}_{n}, \bar{Q}_{n}$ and $R_{n}$, $Q_{n}$ are used to denote potentials of spectral problem (1.2) and that of spectral problem transformed through formula (5.1) respectively, then the relations between them are formulized as follows:

$$
\bar{Q}_{n}=Q_{n}-h_{2}, \bar{R}_{n}=R_{n}+E h_{3},
$$

where $h_{j}, j=1,2,3,4$ are determined by

$$
\begin{aligned}
& h_{j}=\frac{\Delta_{j}}{\Delta}, \Delta=\theta_{n}^{(2)}-\theta_{n}^{(1)}, \Delta_{1}=\lambda_{2} \theta_{n}^{(1)}-\lambda_{1} \theta_{n}^{(2)}, \Delta_{2}=\lambda_{1}-\lambda_{2}, \\
& \Delta_{3}=\left(\lambda_{2}-\lambda_{1}\right) \theta_{n}^{(1)} \theta_{n}^{(2)}, \Delta_{4}=\lambda_{1} \theta_{n}^{(1)}-\lambda_{2} \theta_{n}^{(2)},
\end{aligned}
$$

where $\theta_{n}^{(j)}=\theta_{n}\left(\lambda_{j}\right), j=1,2$ and $\theta_{n}(\lambda)$ is solution of Riccati equation

$$
E \theta_{n}=\frac{R+\theta_{n}}{\lambda+R_{n} Q_{n}+Q_{n} \theta_{n}}, \theta_{n t}=C_{n}+\left(D_{n}-A_{n}\right) \theta_{n}-B \theta_{n}^{2} .
$$

Proof. Transformation $T$ as DT must solve the following equation

$$
\bar{M} H=(E H) M, \bar{M}=\left(\begin{array}{cc}
\lambda+\bar{R}_{n} \bar{Q}_{n} & \bar{Q}_{n} \\
\bar{R}_{n} & 1
\end{array}\right) .
$$

Comparing coefficients of $\lambda^{j}, j=0,1$ in entries of both side yields

$$
\begin{gathered}
\bar{R}_{n} \bar{Q}_{n}-R_{n} Q_{n}=(E-1) h_{1}, \bar{Q}_{n}\left(h_{1} \bar{R}_{n}+h_{3}\right)=\left(Q_{n} E h_{1}+E h_{2}\right) R_{n}, \\
\bar{Q}_{n}=Q_{n}-h_{2}, \bar{Q}_{n}\left(\bar{R}_{n} h_{2}+h_{4}\right)=R_{n}\left(Q_{n} E h_{3}+E h_{4}\right), \\
\bar{R}_{n}=R_{n}+E h_{3}, \bar{R}_{n} E h_{1}+E h_{3}=R_{n}\left(Q_{n} E h_{3}+E h_{4}\right), \\
\bar{R}_{n} h_{2}-Q_{n} E h_{3}=(E-1) h_{4} .
\end{gathered}
$$

Suppose $\lambda_{j}, j=1,2$ are two zeros of $\operatorname{det}(H)$, then components of $H \varphi$ are linear dependent, which means $H \varphi=0$. Thus $h_{j}$ can be determined according to formulas (5.3).

Transformation (5.1) also change (2.1) to time evolution which matches to $\bar{M}$. To justify this assertion, we first consider

$$
V=\left(H_{t}+H N\right) H^{*}, V=\left(\begin{array}{cc}
V_{11} & V_{12} \\
V_{21} & V_{22}
\end{array}\right),
$$

where $H^{*}$ means adjoint matrix of $H$. The expressions of $V_{i j}, i, j=1,2$ are as follows

$$
\begin{gathered}
V_{11}=\left(\lambda+h_{4}\right) h_{1 t}-h_{2 t} h_{3}+\left(\lambda+h_{1}\right)\left(\lambda+h_{4}\right) A_{n}+h_{2}\left(\lambda+h_{4}\right) C_{n}-\left(\lambda+h_{1}\right) h_{3} B_{n}-h_{2} h_{3} D_{n}, \\
V_{12}=\left(\lambda+h_{1}\right) h_{2 t}-h_{1 t} h_{2}-\left(\lambda+h_{1}\right) h_{2} A_{n}-h_{2}^{2} C_{n}+\left(\lambda+h_{1}\right)^{2} B_{n}+\left(\lambda+h_{1}\right) h_{2} D_{n},
\end{gathered}
$$




$$
\begin{gathered}
V_{21}=\left(\lambda+h_{4}\right) h_{3 t}-h_{3} h_{4 t}+\left(\lambda+h_{4}\right) h_{3} A_{n}+\left(\lambda+h_{4}\right)^{2} C_{n}-h_{3}^{2} B_{n}-h_{3}\left(\lambda+h_{4}\right) D_{n}, \\
V_{22}=-h_{2} h_{3 t}+\left(\lambda+h_{1}\right) h_{4 t}-h_{2} h_{3} A_{n}-h_{2}\left(\lambda+h_{4}\right) C_{n}+\left(\lambda+h_{1}\right) h_{3} B_{n}+\left(\lambda+h_{1}\right)\left(\lambda+h_{4}\right) D_{n} .
\end{gathered}
$$

We will prove that $\lambda_{j}, j=1,2$ are zeros of $V_{i j}, i, j=1,2$ and $V_{11}, V_{22}$ and $V_{12}, V_{21}$ are polynomials of $\lambda$ with degree $k+3$ and $k+2$ respectively. This assures entries of matrix $\tilde{N}=\left(H_{t}+H N\right) H^{-1}$ have the same degrees as that of $N$. Assume asymptotic condition

$$
N=\left(\begin{array}{cc}
A_{n} & B_{n} \\
C_{n} & D_{n}
\end{array}\right) \rightarrow\left(\begin{array}{cc}
\frac{\lambda^{k+1}}{2} & 0 \\
0 & -\frac{\lambda^{k+1}}{2}
\end{array}\right), \text { when }|n| \rightarrow \infty,
$$

and $h_{2}, h_{3} \rightarrow 0$ when $|n| \rightarrow \infty$ we will find that $\tilde{N}=\left(H_{t}+H N\right) H^{-1}$ has the same asymptotic behavior. Therefore, because of $\tilde{N}, \bar{N}$ also satisfy the same different equation, we say $\tilde{N}=\bar{N}$, i.e., transformation (5.1) change (2.1) into time evolution matching to $\bar{M}$.

Now we deal with $V_{11}$ as an example. First of all, referring to the fact that $A_{n}, D_{n}$ and $B_{n}, C_{n}$ are polynomials of $\lambda$ with degree $k+1$ and $k$, it is ease to know $V_{11}$ is polynomial of $\lambda$ with degree $k+3$. Secondly, according to the definition of $h_{j}, j=1,2,3,4$ we work out

$$
h_{1 t}=\frac{h_{2}\left(\theta_{n}^{(1)} \theta_{n t}^{(2)}-\theta_{n}^{(2)} \theta_{n t}^{(1)}\right)}{\theta_{n}^{(2)}-\theta_{n}^{(1)}}, h_{2 t}=\frac{h_{2}\left(\theta_{n t}^{(1)}-\theta_{n t}^{(2)}\right)}{\theta_{n}^{(2)}-\theta_{n}^{(1)}} .
$$

Substituting them and (5.4) into $V_{11}$ gives rise to

$$
\begin{aligned}
V_{11}\left(\lambda_{j}\right)= & \frac{1}{\theta_{n}^{(2)}-\theta_{n}^{(1)}}\left[h_{2}\left(\lambda_{j}-\lambda_{1}\right) \theta_{n}^{(1)}\left(C_{n}^{(2)}-2 A_{n}^{(2)} \theta_{n}^{(2)}-B_{n}^{(2)} \theta_{n}^{(2) 2}\right)\right. \\
& -h_{2}\left(\lambda_{j}-\lambda_{2}\right) \theta_{n}^{(2)}\left(C_{n}^{(1)}-2 A_{n}^{(1)} \theta_{n}^{(1)}-B_{n}^{(1)} \theta_{n}^{(1) 2}\right)+2\left(\lambda_{1}-\lambda_{2}\right) h_{3} A_{n}^{(j)} \\
& \left.+\left(\lambda_{1}-\lambda_{2}\right)\left(\lambda_{j}+h_{4}\right) C_{n}^{(j)}+\left(\lambda_{1}-\lambda_{2}\right)\left(\lambda_{j}+h_{1}\right) \theta_{n}^{(1)} \theta_{n}^{(2)} B_{n}^{(j)}\right], \quad j=1,2 .
\end{aligned}
$$

It is not difficult to check that $V_{11}\left(\lambda_{j}\right)=0, j=1,2$. Noticing that $\operatorname{det}(H)=\left(\lambda-\lambda_{1}\right)\left(\lambda-\lambda_{2}\right)$, we may say that $\tilde{A}_{n}$ in

$$
\tilde{N}=\left(\begin{array}{cc}
\tilde{A}_{n} & \tilde{B}_{n} \\
\tilde{C}_{n} & \tilde{D}_{n}
\end{array}\right)
$$

is $k+1$ power polynomial of $\lambda$. As for asymptotic behavior of $V_{11}$, obviously, $\left|\lambda_{1}\right|>1>\left|\lambda_{2}\right|$ make $h_{2}, h_{3} \rightarrow 0$ as $|n| \rightarrow \infty$ hold. In the mean time, $h_{1}, h_{4}$ tend to $-\lambda_{1},-\lambda_{2}$. These results assure $\tilde{A}_{n} \rightarrow \frac{\lambda^{k+1}}{2}$. When it comes to $V_{12}, V_{21}, V_{22}$, the proof is similar, we do not repeat it. Now we finish the proof that (5.1) is a Darboux transformation of Ragnisco-Tu hierarchy.

As an application we present a exact solution to Ragnisco-Tu Equation (1.1). Starting from seed solution $R=Q=0$, we first obtain a solution to Riccati Equations (5.4)

$$
\theta_{n}=\lambda^{-n} \mathrm{e}^{-\lambda^{k}}
$$

Then according to Lemma 5.1, a solution to Ragnisco-Tu can be calculated out as follows

$$
\bar{Q}_{n}=-\frac{\lambda_{1}-\lambda_{2}}{\lambda_{2}^{-n} \mathrm{e}^{-\lambda_{2} t}-\lambda_{1}^{-n} \mathrm{e}^{-\lambda_{1} t}}, \bar{R}_{n}=-\frac{\left(\lambda_{1}-\lambda_{2}\right)\left(\lambda_{1} \lambda_{2}\right)^{-n-1} \mathrm{e}^{-\left(\lambda_{1}+\lambda_{2}\right) t}}{\lambda_{2}^{-n} \mathrm{e}^{-\lambda_{2} t}-\lambda_{1}^{-n} \mathrm{e}^{-\lambda_{1} t}},
$$

\subsection{Darboux Transformation to Generalized Ragnisco-Tu Equation}

From gauge transformation $\bar{\varphi}=T \bar{\psi}, \varphi=T \psi$ and DT of Ragnisco-Tu equation $\bar{\varphi}=H \varphi$, we find relation between $\bar{\psi}$ and $\psi$ : 


$$
\bar{\psi}=\bar{T}^{-1} H T \psi,
$$

which forms DT of generalized Ragnisco-Tu equation. Matrix $\bar{T} H T^{-1}$ admits the following form

$$
\bar{T} H T^{-1}=\left(\begin{array}{cc}
\left(\lambda+h_{1}\right) E^{-1} \frac{\mu_{n}}{\bar{\mu}_{n}}-\bar{\mu}_{n} \bar{q}_{n} h_{3} E^{-1} \mu_{n} & \left(\lambda+h_{1}\right) \frac{\mu_{n} q_{n}}{E^{-1} \bar{\mu}_{n}}-\left(\lambda+h_{4}\right) \frac{\bar{q}_{n} \bar{\mu}_{n}}{E^{-1} \mu_{n}}+\frac{h_{2}}{E^{-1} \mu_{n} \bar{\mu}_{n}}-h_{3} q_{n} \bar{q}_{n} \mu_{n} \bar{\mu}_{n} \\
h_{3} E^{1} \mu_{n} \bar{\mu}_{n} & \left(\lambda+h_{4}\right) E^{-1} \frac{\bar{\mu}_{n}}{\mu_{n}}+h_{3} q_{n} \mu_{n} E^{-1} \bar{\mu}_{n}
\end{array}\right) .
$$

We can adopt simple notation to write it

$$
\bar{\psi}=P \psi, \quad P=\left(\begin{array}{cc}
p_{11}^{(1)} \lambda+p_{11}^{(0)} & p_{12}^{(1)} \lambda+p_{12}^{(0)} \\
p_{21}^{(0)} & p_{22}^{(1)} \lambda+p_{22}^{(0)}
\end{array}\right),
$$

where

$$
\begin{gathered}
p_{11}^{(1)}=E^{-1} \frac{\mu_{n}}{\bar{\mu}_{n}}, p_{11}^{(0)}=h_{1} E^{-1} \frac{\mu_{n}}{\bar{\mu}_{n}}-\bar{\mu}_{n} \bar{q}_{n} h_{3} E^{-1} \mu_{n}, p_{12}^{(1)}=\frac{\mu_{n} q_{n}}{E^{-1} \bar{\mu}_{n}}-\frac{\bar{q}_{n} \bar{\mu}_{n}}{E^{-1} \mu_{n}}, \\
p_{12}^{(0)}=h_{1} \frac{\mu_{n} q_{n}}{E^{-1} \bar{\mu}_{n}}-h_{4} \frac{\bar{q}_{n} \bar{\mu}_{n}}{E^{-1} \mu_{n}}+\frac{h_{2}}{E^{-1} \mu_{n} \bar{\mu}_{n}}-h_{3} q_{n} \bar{q}_{n} \mu_{n} \bar{\mu}_{n}, \\
p_{21}^{(0)}=h_{3} E^{1} \mu_{n} \bar{\mu}_{n}, p_{22}^{(1)}=E^{-1} \frac{\bar{\mu}_{n}}{\mu_{n}}, p_{22}^{(0)}=h_{4} E^{-1} \frac{\bar{\mu}_{n}}{\mu_{n}}+h_{3} q_{n} \mu_{n} E^{-1} \bar{\mu}_{n},
\end{gathered}
$$

As a DT of generalized Ragnisco-Tu equation, $P$ should satisfy

$$
\bar{M}^{\prime} P=(E P) M^{\prime},
$$

where

$$
\bar{M}^{\prime}=\left(\begin{array}{cc}
\lambda\left(1+\bar{q}_{n} \bar{r}_{n}\right)+\bar{q}_{n} \bar{r}_{n} & (\lambda+1) \bar{q}_{n} \\
\bar{r}_{n} & 1
\end{array}\right) .
$$

From this expression, we can draw the following equalities

$$
\begin{gathered}
\left(1+\bar{q}_{n} \bar{r}_{n}\right) p_{11}^{(1)}=\left(1+q_{n} r_{n}\right) E p_{11}^{(1)}, \\
p_{11}^{(0)}+\bar{q}_{n} \bar{r}_{n} p_{11}^{(1)}+\bar{q}_{n}\left(\bar{r}_{n} p_{11}^{(0)}+p_{21}^{(0)}\right)=E p_{11}^{(0)}+q_{n} r_{n} E p_{11}^{(1)}+r_{n}\left(q_{n} E p_{11}^{(1)}+E p_{12}^{(1)}\right), \\
\bar{q}_{n}\left(\bar{r}_{n} p_{11}^{(0)}+p_{21}^{(0)}\right)=r_{n}\left(q_{n} E p_{11}^{(0)}+E p_{12}^{(0)}\right), \\
p_{12}^{(1)}+\bar{q}_{n}\left(\bar{r}_{n} p_{12}^{(1)}+p_{22}^{(1)}\right)=q_{n} E p_{12}^{(1)}, \\
p_{12}^{(0)}+\bar{q}_{n}\left(\bar{r}_{n} p_{12}^{(1)}+p_{22}^{(1)}\right)+\bar{q}_{n}\left(\bar{r}_{n} p_{12}^{(0)}+p_{22}^{(0)}\right)=q_{n}\left(E p_{11}^{(0)}+E p_{11}^{(1)}\right)+E p_{12}^{(1)}, \\
\bar{q}_{n}\left(\bar{r}_{n} p_{12}^{(0)}+p_{22}^{(0)}\right)=q_{n} E p_{11}^{(0)}+E p_{12}^{(0)}, \\
\bar{r}_{n} p_{11}^{(1)}=\left(1+q_{n} r_{n}\right) E p_{21}^{(0)}+r_{n} E p_{22}^{(1)}, \\
\bar{r}_{n} p_{11}^{(0)}+p_{21}^{(0)}=r_{n}\left(q_{n} E p_{21}^{(0)}+E p_{22}^{(0)}\right), \\
\bar{r}_{n} p_{12}^{(1)}+p_{22}^{(1)}=q_{n} E p_{21}^{(0)}+E p_{22}^{(1)}, \\
\bar{r}_{n} p_{12}^{(0)}+p_{22}^{(0)}=q_{n} E p_{21}^{(0)}+E p_{22}^{(0)} .
\end{gathered}
$$

The acquisition of solution of them must be combined with relation exhibited in Darboux matrix (5.13). Here we do not consider general formula of solution but present a special solution related to $q_{n}=r_{n}=0$. 
Notice that $\operatorname{det}(H)=\left(\lambda-\lambda_{1}\right)\left(\lambda-\lambda_{2}\right)$ and the definitions of $h_{j}, j=1,2,3,4$ (5.3), we can find some simple relations easily

$$
h_{1} E h_{4}=\lambda_{1} \lambda_{2}, h_{3} E h_{4}=-\lambda_{1} \lambda_{2} E h_{3}, p_{11}^{(0)} p_{22}^{(0)}-p_{12}^{(0)} p_{21}^{(0)}=\lambda_{1} \lambda_{2} .
$$

When seed solution $q_{n}=r_{n}=0$ is substituted into (5.16a)-(5.19b) we find following relations

$$
p_{11}^{(0)}=p_{11}^{(1)}+1, p_{12}^{(0)}=p_{12}^{(1)} \text {. }
$$

These equalities produce

$$
E^{-1} \bar{\mu}_{n}=h_{1}-1-\frac{h_{3}}{E h_{3}} \bar{q}_{n} \bar{r}_{n}
$$

and

$$
\bar{q}_{n} \bar{r}_{n}=\frac{h_{2} E h_{3}}{h_{4}-1} .
$$

Thus we get

$$
E^{-1} \bar{\mu}_{n}=\frac{\left(\lambda_{1}+1\right)\left(\lambda_{2}+1\right)}{h_{4}-1}
$$

and form (5.17c), we obtain

$$
\bar{q}_{n}=\frac{E h_{4}-1}{\left(\lambda_{1}+1\right)\left(\lambda_{2}+1\right) \Delta} .
$$

Substituting it into (5.21), $\bar{r}_{n}$ is figured out

$$
\bar{r}_{n}=\frac{\left(\lambda_{1}+1\right)\left(\lambda_{2}+1\right)\left(h_{2} E h_{3}\right) \Delta}{\left(h_{4}-1\right)\left(E h_{4}-1\right)} .
$$

\section{Conclusion}

We propose a lattice equation hierarchy related to Rangnisco-Tu hierarchy (generalized RT equation) and prove that it is equivalent to Rangnisco-Tu hierarchy itself. The transfer operator of two hierarchies is obtained. As an application of gauge transformation, we obtain a Darboux transformation of generalized RT equation and acquire an exact solution of this equation.

\section{Acknowledgements}

The authors feel grateful to pertinent opinions of reviewer and careful work of editors.

\section{References}

[1] Chen, D.Y. (2006) Introduction of Soliton. Science Press, Beijing. (In Chinese)

[2] Chen, D.Y. and Zeng, Y.B. (1985) The Transformation Operator of Nonlinear Evolution Equations I. Chinese Annals of Mathematics, 6B, 71-82.

[3] Chen, D.Y. and Zeng, Y.B. (1985) The Transformation Operator of Nonlinear Evolution Equations III. Acta Mathematica, 28, 161-173. (In Chinese)

[4] Chen, D.Y. and Li, Y.S. (1986) The Transformation Operator of Nonlinear Evolution Equations IV. Acta Mathematica, 29, 127-134. (In Chinese)

[5] Chen, D.Y. and Li, Y.S. (1986) The Transformation Operator of Nonlinear Evolution Equations V. Acta Mathematica Sinica, New Series, 2, 343-356. http://dx.doi.org/10.1007/BF02564935

[6] Chau, L.L., Shaw, J.C. and Yen, H.C. (1992) Solving the KP Hierarchy by Gauge Transformations. Communications in Theoretical Physics, 149, 263-278. http://dx.doi.org/10.1007/bf02097625

[7] Zhang, H.W., Tu, G.Z., et al. (1991) Symmetries, Conserved Quantities, and Hierarchies for Some Lattice Systems 
with Soliton Structure. Journal of Mathematical Physics, 32, 1908-1918. http://dx.doi.org/10.1063/1.529205

[8] Zeng, Y.B. (1998) Restried Flows of a Hierarchy of Integrable Discrete Systems. Acta Mathematicae Applicatae Sinica, 14, 176-184. http://dx.doi.org/10.1007/BF02677424

[9] Liu, Y.Q. and Chen, D.Y. (2011) The Exact Solutions to a Ragnisco-Tu Hierarchy with Self-Consistent Sources. Nonlinear Analysis, 74, 5223-5237. http://dx.doi.org/10.1016/j.na.2011.04.021

[10] Xu, X.X. (2010) An Integrable Coupling Family of Merola-Ragnisco-Tu Lattice Systems, Its Hamiltonian Structure and Related Nonisospectral Integrable Lattice Family. Physics Letters A, 374, 401-410. http://dx.doi.org/10.1016/j.physleta.2009.11.012

[11] Zeng, X. and Geng, X.G. (2014) Algebro-Geometric Solutions of the Discrete Ragnisco-Tu Hierarchy. Reports on Mathematical Physics, 73, 17-48. http://dx.doi.org/10.1016/S0034-4877(14)60030-6

[12] Xu, X.X. (2015) Solving an Integrable Coupling System of Merola-Ragnisco-Tu Lattice Equation by Darboux Transformation of Lax Pair. Communications in Nonlinear Science and Numerical Simulation, 23, 192-201. http://dx.doi.org/10.1016/j.cnsns.2014.11.002

[13] Xu, X.X., Yang, H.X. and Sun, Y.P. (2006) Darboux Transformation of the Modified Toda Lattice Equation. Modern Physics Letters B, 20, 641-648. http://dx.doi.org/10.1142/S0217984906011025

[14] Hon, Y.C. and Fan, E.G. (2009) Quasi-Periodic Solutions for Modified Toda Lattice Equation. Chaos, Solitons and Fractals, 40, 1297-1308. http://dx.doi.org/10.1016/j.chaos.2007.09.008

[15] Spiridonov, V. and Zhedanov, A. (1995) Discrete Darboux Transformations, the Discrete-Time Toda Lattice, and the Askey-Wilson Polynomials. Methods and Applications of Analysis, 2, 369-398.

[16] Fan, E.G. (2001) Explicit $N$-Fold Darboux Transformations and Soliton Solutions for Nonlinear Derivative Schrödinger Equations. Communications in Theoretical Physics, 35, 651-656. http://dx.doi.org/10.1088/0253-6102/35/6/651

[17] Zeng, Y.B., Shao, Y.J. and Ma, W.X. (2002) Integral-Type Darboux Transformations for the mKdV Hierarchy with Self-Consistent Sources. Communications in Theoretical Physics, 38, 641-648. http://dx.doi.org/10.1088/0253-6102/38/6/641

[18] He, J.S., Zhang, L., Chen, Y. and Li, Y.S. (2006) Determinant Representation of Darboux Transformation for the AKNS System. Science in China Series A: Mathematics, 49, 1867-1878. http://dx.doi.org/10.1007/s11425-006-2025-1

[19] Zhou, Z.X. (2007) Darboux Transformations for Some Two Dimensional Affine Toda Equations. The International Congress of Chinese Mathematicians (ICCM), III, 405-416.

[20] Wang, L., Gao, Y.T., Gai, X.L., Yu, X. and Sun, Z.Y. (2010) Vadermonde-Type Odd-Soliton Solutions for the Whitham-Broer-Kaup Model in the Shallow Water Small-Amplitude Regime. Journal of Nonlinear Mathematical Physics, 17, 197-211. http://dx.doi.org/10.1142/S1402925110000714

[21] Geng, X.G. and He, G.L. (2010) Darboux Transformation and Explicit Solutions for the Satsuma-Hirota Coupled Equation. Applied Mathematics and Computation, 216, 2628-2634. http://dx.doi.org/10.1016/j.amc.2010.03.107

[22] Xu, G.H. (2014) N-Fold Darboux Transformation of the Jaulent-Miodek Equation. Applied Mathematics, 5, $2657-2663$. http://dx.doi.org/10.4236/am.2014.517254 